\title{
Outcome analysis of intussusception in fifty children at a tertiary centre in Mumbai
}

\author{
Tiwari $\mathbf{C}^{\mathbf{1}}$, Sandlas $\mathbf{G}^{2}$, Jayaswal $\mathrm{S}^{3}$, Shah $\mathrm{H}^{4}$ \\ ${ }^{1}$ Dr Charu Tiwari, MCh Resident, Dept of Paediatric Surgery, TNMC \& BYL Nair Hospital, Mumbai, Maharashtra, ${ }^{2}$ Dr \\ Gursev Sandlas, Assistant Professor, ${ }^{3}$ Dr Shalika Jayaswal, Associate Professor, ${ }^{4}$ Dr Hemanshi Shah, Professor and \\ Head. All are affiliated with TNMC \& BYL Nair Hospital, Mumbai, Maharashtra, India
}

Address for correspondence: Dr Hemanshi Shah, E-Mail ID: hemanshisshah@ gmail.com

\begin{abstract}
Background: Intussusception is a common cause of obstruction in Paediatric patients. Recognizing and treating this condition rapidly is important to prevent potentially fatal complications. Methods: Fifty consecutive patients of intussusception were analyzed with respect to age, presenting symptoms with duration, laboratory and radiological findings, type of management, duration of hospital stay and follow-up. Results: The median age at presentation was 11 months. Patients presenting between 3 months and 2 years of age are more likely to require operative intervention. The most common presenting symptoms were abdominal pain and vomiting. The diagnosis of intussusception was made by Ultrasonography and a trial of non-operative intervention was given first by in the form of USG guided hydrostatic reduction. Most of the patients required two or lesser attempts of reduction and were thus managed non-operatively. Those who did not respond to 2 or more attempts of hydrostatic reduction required operative intervention and all of these had some or the other lead point. Eight out of 11 operated patients $(72.72 \%)$ required resection for the gangrenous bowel segment. The most common type of intussusception was ileo-colic and most of them were idiopathic. Lead points were found in all the patients who were operated; the most common being enlarged mesenteric lymph nodes. Conclusion: Children presenting between 3 months to 2 years of age, with no palpable abdominal lump or passage of red currant jelly-like stools as symptoms and getting reduced hydrostatically at one or two attempts mostly have idiopathic intussusception and such patients won't require operative intervention.
\end{abstract}

Keywords: Intussusception, Red Currant Jelly Stools, Hydrostatic Reduction, Operative Intervention.

\section{Introduction}

Intussusception is the invagination of one part of the bowel into another. It is one of the most frequent causes of acute bowel obstruction in infants and toddlers and the second most common cause of acute abdominal pain in preschool children after constipation $[1,2]$. Recognizing and treating this condition rapidly is important to prevent potentially fatal complications [3]. The diagnosis is usually based on clinical features [4], which may be quite challenging. This study analyses 50 patients of intussusception with respect to age, presenting symptoms with duration, laboratory and radiological findings, type of management, duration of hospital stay and follow-up as per set proforma.

Manuscript received: $5^{\text {th }}$ Sept 2015

Reviewed: $10^{\text {th }}$ Sept 2015

Author Corrected; $18^{\text {th }}$ Sept 2015

Accepted for Publication: $2^{\text {nd }}$ Oct 2015

\section{Materials and Method}

This is a prospective observational non-randomized study done on fifty consecutive children who presented with clinical suspicion of intussusception. Inclusion criteria was age less than 12 years and isolated abdominal complaints suggestive of intussusception like abdominal pain, vomiting, abdominal lump, red currant jelly stools, constipation, etc. Patients more than 12 years of age and with history of previous operative procedure and blunt trauma to abdomen were excluded. These patients were admitted in the Paediatric Surgery ward in TNMC and BYL Nair Hospital. After laboratory investigations, an erect abdominal X-Ray was done for presence of complete obstruction and/or pneumoperitoneum-these patients would have warranted immediate surgical intervention. At Ultrasonography (USG), once the diagnosis of 
intussusception was confirmed, USG guided hydrostatic reduction was attempted. The "rule of threes" (three attempts, each of three minutes duration and with the saline bottles at three feet height) was followed [4]. There was a gap of at least 6 hours between two reductions and in the meantime the patients received intravenous fluids, intravenous antibiotics, antispasmodics and steroids in the ward. The second and third attempts were tried only after confirming the viability of the bowel on Ultrasound. Those patients who did not respond to three attempts of reduction were taken for operative intervention.

\section{Results}

A total of 50 consecutive patients were included in this study. Non-operative intervention in the form of hydrostatic reduction was successful in 38 patients $(76 \%)$. Remaining 12 patients $(24 \%)$ required operative intervention. The ones that required operative intervention were those with delayed presentation (more than 48 hours).

They also had some evident lead point at surgery and 8 out of these 11 operated patients (72.72\%) had gangrenous bowel necessitating resection. One patient, though responding to hydrostatic reduction, had multiple episodes of recurrent intussusception and was operated later.

The median age at presentation was 11 months with a range of 3 months to 144 months. Out of the 50 patients, 2 patients were less than 3 months of age and half of them responded to hydrostatic reduction and 15 patients were more than 2 years of age out of which 10 patients $(66.66 \%)$ responded to hydrostatic reduction.

Out of the total 50 patients, 37 had symptoms of less than 48 hours and only 2 patients (5.4\%) among them required operative intervention; 13 had symptom duration of more than 48 hours of which 9 patients $(69.23 \%)$ required operative intervention.

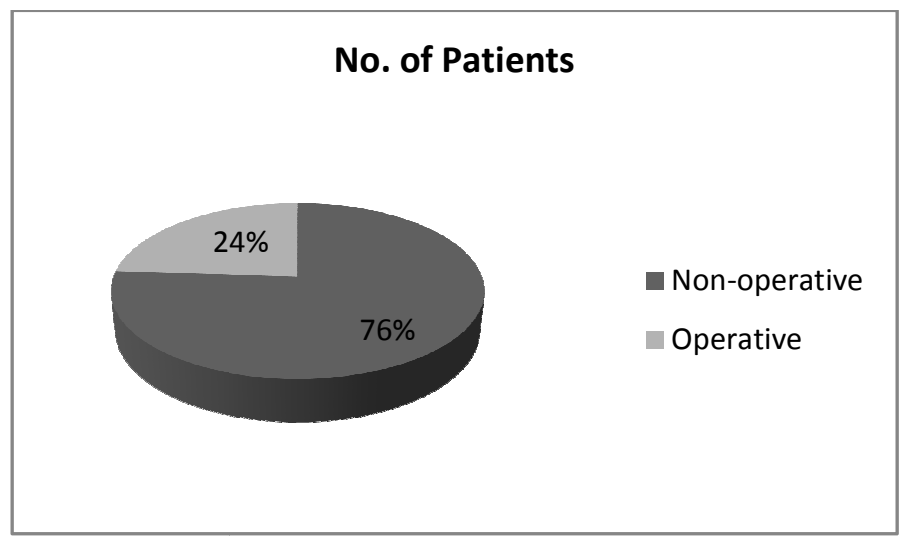

Figure 1: Pie Chart depicting percentage of patients managed Non-operatively and Operatively.

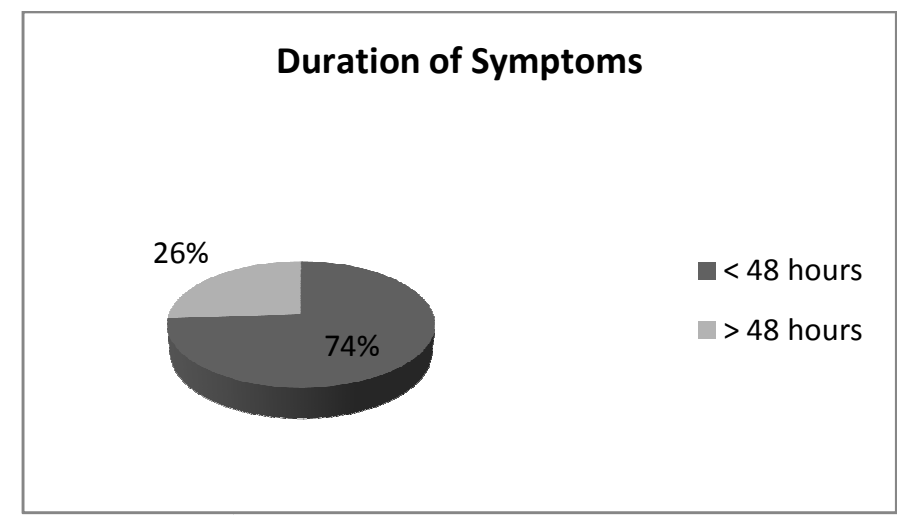

Figure 2: Pie Chart depicting percentage of patients with Early ( $<48$ hours) and Delayed Presentation ( $>48$ hours). 


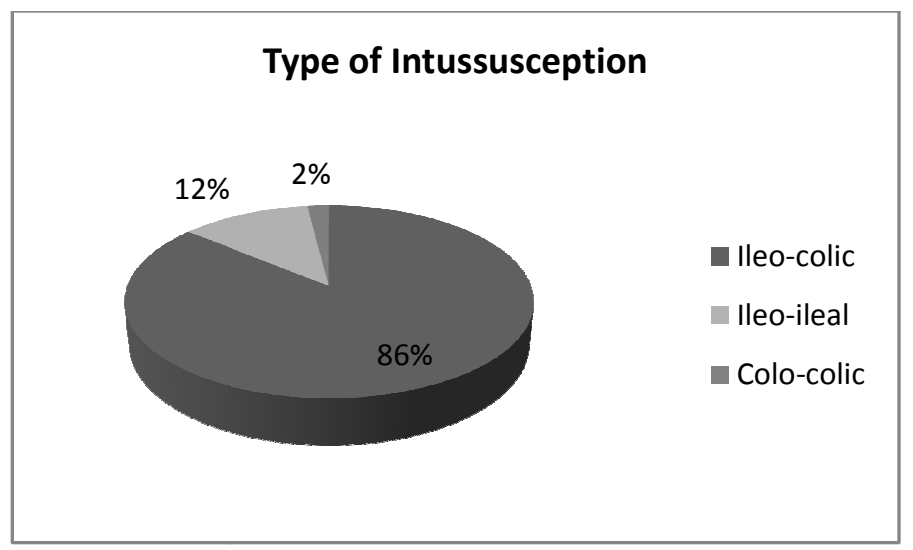

Figure 3: Pie Chart depicting the percentages of various Types of Intussusception.

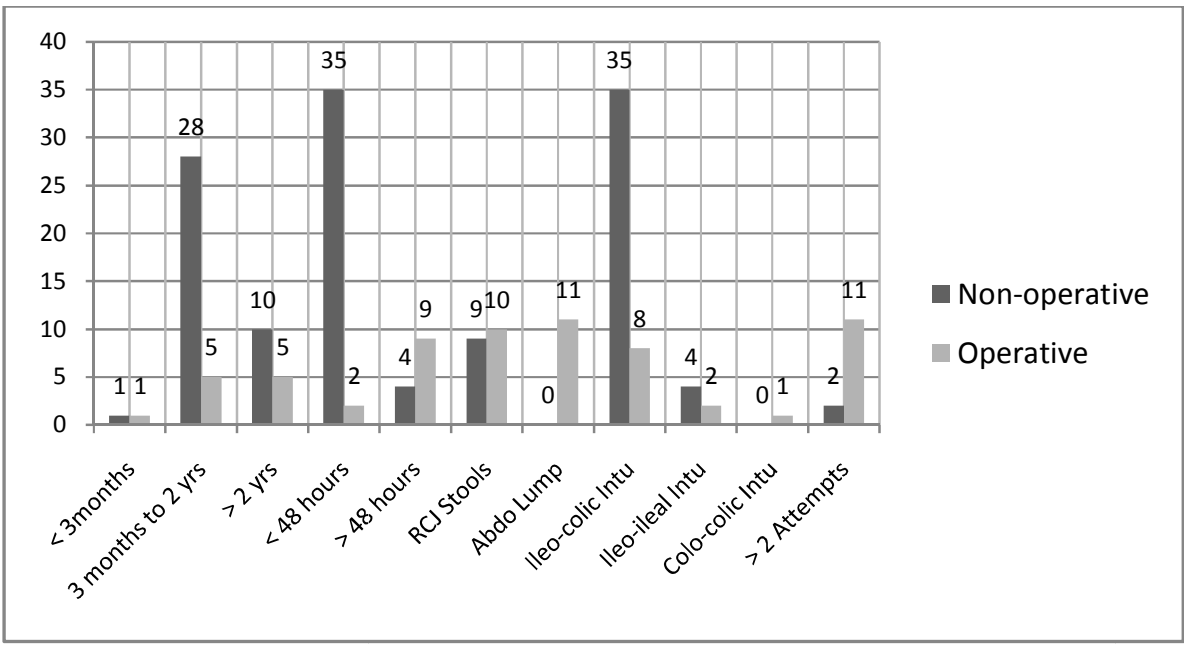

Figure 4: Bar Chart depicting Non-operative v/s Operative Intervention with respect to presentation, symptoms, types of Intussusception and attempts required for reduction.

The most common symptoms and signs were abdominal pain seen in 49 patients (98\%), vomiting in 46 patients (92\%) and tachycardia present in 40 patients $(80 \%)$. Red currant jelly-like stools were present in 19 patients $(38 \%)$ and a palpable abdominal lump was present in 11 patients $(22 \%)$. Out of the 19 patients presenting with red currant jelly like stools, only 9 patients (47.3\%) responded to hydrostatic reduction. 11 patients having a palpable abdominal lump not responded to hydrostatic reduction. Thus, patients with palpable abdominal lump and red currant jelly-like stools are more likely to require surgery. Weaning was associated in 19 patients from which 17 patients $(89.5 \%)$ responded to hydrostatic reduction.

The WBC Count was normal in 49 patients out of which 39 patients $(79.6 \%)$ responded to hydrostatic reduction. Only 1 patient had counts more than 14,000/cu.mm and required laparotomy with bowel resection for gangrene. Air-fluid levels were seen in 5 patients and none of them responded to hydrostatic reduction. (4 had delayed presentation)

Ileo-colic intussusception was the most common type of intussusception diagnosed on USG; it was seen in 43 patients out of which 35 patients $(81.3 \%)$ responded to hydrostatic reduction. Ileo-ileal intussusception was seen in 6 patients and 4 of them $(66.66 \%)$ responded to hydrostatic reduction. One patient had colo-colic intussusception but did not respond to hydrostatic reduction and required laparotomy. However he had delayed presentation and did not respond to three attempts of hydrostatic reduction.

Intussusception was reduced at the first 2 attempts of hydrostatic reduction in 37 patients $(74 \%)$. Of the 
remaining 13 patients, 2 responded to hydrostatic reduction at the third attempt. The remaining 11 patients required operative intervention.

Ultrasonography detected enlarged mesenteric lymph nodes in 15 patients and 2 patients had thickened colon. Both the patients with thickened colon responded to hydrostatic reduction. Seven patients with enlarged mesenteric lymph nodes responded to hydrostatic reduction. At laparotomy, 8 patients had enlarged mesenteric lymph nodes as the lead point and 5 of them had gangrenous bowel requiring resection. Meckel's Diverticulum was the lead point in 2 patients and both required resection for gangrenous bowel. Hypertrophic Peyer's patches were the lead point in 2 patients of which 1 had gangrene of the bowel and required resection.

Operative intervention was required in 12 patients (24\%) of which 9 patients had delayed presentation of more than 48 hours; 11 patients had palpable abdominal lump and 10 patients had red currant jelly-like stools on examination. Eleven patients did not respond to third attempt of reduction and 8 patients had gangrene of the bowel requiring resection. One patient was operated because of multiple episodes of recurrent intussusception.

Post-operative course was uneventful in all the patients except for a delayed perforation of the transverse colon in one patient requiring colostomy and superficial surgical site infection in another. The hospital stay was less than 4 days in 38 patients who were managed conservatively; 11 operated patients had a hospital stay of 4 to 14 day; 1 patient had hospital stay of more than 14 days due to postoperative complications.

\section{Discussion}

This study analyses the presentation and outcome of intussusception in urban Indian children. The median age at presentation was 11 months which correlates well with the age at presentation in most of the other studies [2] and also with the Western data [5]. Patients presenting in less than 3 months of age and more than 2 years of age are more likely to require operative intervention. The most common presenting symptoms were abdominal pain and vomiting. Presence of a lump in abdomen and red currant jelly-like stools were less common symptoms and were usually present in children with late presentation and all patients with these findings required surgery and had a prolonged hospital stay.
The diagnosis of intussusception was made by Ultrasonography and a trial of non-operative intervention was given first by in the form of USG guided hydrostatic reduction with saline. The hydrostatic reduction is as good as the pneumatic reduction done in western countries. Multiple studies done previously not reached a definitive conclusion regarding the superiority of one type of reduction over the other. Each technique has its own risks and benefits $[6,7]$.

Most of the patients responded to the first two attempts of reduction and were thus managed non-operatively. Those who did not respond to third attempt of hydrostatic reduction required operative intervention and all of these had some or the other lead point and 8 out of 12 operated patients $(66.66 \%)$ required resection for the gangrenous bowel segment. These were those who had delayed presentation.

The most common type of intussusception was found to be ileo-colic which also correlates well with that reported in other studies [8] and most of them responded to non-operative management by hydrostatic reduction.

Most of the intussusceptions (58\%) were idiopathic and all of these idiopathic intussusceptions got reduced by hydrostatic reduction. Lead points were found in all the patients who were operated; the most common being enlarged mesenteric lymph nodes found in 8 of the 12 operated patients $(66.66 \%)$. In 2 of the operated patients with ileo-ileal intussusception, Meckel's Diverticulum was the lead point and both of them were more than 5 years of age and required resection of the gangrenous segment of small bowel. This finding is little different from that of the Western data where lead points are detected in lesser patients at surgery and Meckel's Diverticulum or malignancy are the causes [9].

The operative rate of 12 out of total 50 patients $(24 \%)$ is less than that reported in other studies $[5,8]$. The bowel resection rate of 8 out of 12 patients $(66.66 \%)$ is more than the widely varied rates reported in the literature $[10,11]$. This points towards the fact that most of the patients in our setting present late.

The complication rate of 2 out of 50 patients $(4 \%)$ is lesser than that reported in the Western data [10, 12 and 13]. Only one patient managed conservatively initially had recurrent episodes of intussusception and was operated upon later. There was no mortality in this study. 


\section{Conclusion}

To sum up, if the child presents early and is between 3 months to 2 years of age, has no palpable abdominal lump and passage of red currant jelly-like stools as symptoms and gets reduced hydrostatically at one or two attempts, then the intussusception is mostly idiopathic and such patients won't require operative intervention and are likely to be discharged early from the hospital.

However, further ongoing prospective data collection is underway to capture a larger cohort for better statistical analysis.

\section{Funding: None}

Conflict of Interest: None

Permission from IRB: Yes

\section{References}

1. Fallat ME. Intussusception. In: Ashcraft KW, editor. Pediatric Surgery. 3 rd ed. W.B. Saunders Company Philadelphia, Pennsylvania; 2000. p. 518-26.

2. Paul M. Columbani and Stephen Scolz: Paediatric Surgery seventh edition. P. 1093-1110.

3. Young DG. Intussusception. in: O'Neil JA Jr, Rowe MI, Grosfeld JL, Fonkalsrud EW, Coran AG, editors. Pediatric Surgery. 5th ed. Mosby, New York, USA. 1998. p. 1185-98.

4.DENNISON WM. Acute intussusception in infancy and childhood. Glasgow Med J. 1948 Mar;29(3):71-80.

5. Jen HC, Shew SB. The impact of hospital type and experience on the operative utilization in pediatric intussusception: a nationwide study. J Pediatr Surg. 2009 Jan;44(1):241-6. doi: 10.1016/j.jpedsurg.2008.10.050.
6. Daneman A, Navarro O. Intussusception. Part 2: An update on the evolution of management. Pediatr Radiol. 2004 Feb;34(2):97-108; quiz 187. Epub 2003 Nov 21.

7. Katz M, Phelan E, Carlin JB, Beasley SW. Gas enema for the reduction of intussusceptions: relation ship between clinical sign \& symptoms andoutcome. AJR Am J Roentgenol. 1993 Feb;160(2):363-6.

8. Bratton SL, Haberkern CM, Waldhausen JH, Sawin RS, Allison JW. Intussusception: hospital size and risk of surgery. Pediatrics. 2001 Feb;107(2):299-303.

9. Marinis A, Yiallourou A, Samanides L, Dafnios $\mathrm{N}$, Anastasopoulos G, Vassiliou I, Theodosopoulos T. Intussusception of the bowel in adults: a review. World J Gastroenterol. 2009 Jan 28;15(4):407-11.

10. Saxena AK, Höllwarth ME. Factors influencing management and comparison of outcomes in paediatric intussusceptions. Acta Paediatr. 2007 Aug;96(8):1199202. Epub 2007 Jun 21.

11. Fraser JD, Aguayo P, Ho B, Sharp SW, Ostlie DJ, Holcomb GW 3rd, St Peter SD. Laparoscopic management of intussusception in pediatric patients. J Laparoendosc Adv Surg Tech A. 2009 Aug;19(4):5635. doi: 10.1089/lap.2009.0117.

12.Kaiser AD, Applegate KE, Ladd AP. Current success in the treatment of intussusception in children. Surgery. 2007 Oct;142(4):469-75; discussion 475-7.

13.Kodikara H, Lynch A, Morreau P, Vogel S. Tenyear review of intussusception at Starship Hospital: 1998-2007. N Z Med J. 2010 Oct 15;123(1324):32-40.

\section{How to cite this article?}

Tiwari C, Sandlas G, Jayaswal S, Shah H. Outcome analysis of intussusception in fifty children at a tertiary centre in Mumbai. Pediatr Rev: Int J Pediatr Res 2015;2(3):25-29. doi: 10.17511/ijpr.2015.i03.05 